\title{
Investigation of X-ray transient CI Cam as an unique star with the $\mathrm{B}[\mathrm{e}]$ phenomenon
}

\author{
Elena A. Barsukova ${ }^{1}$, Alexandr N. Burenkov ${ }^{1}$, \\ Valentina G. Klochkova ${ }^{1}$, Vitalij P. Goranskij ${ }^{2}$, Nataly V. Metlova ${ }^{2}$, \\ Peter Kroll ${ }^{3}$ and Anatoly S. Miroshnichenko ${ }^{4}$

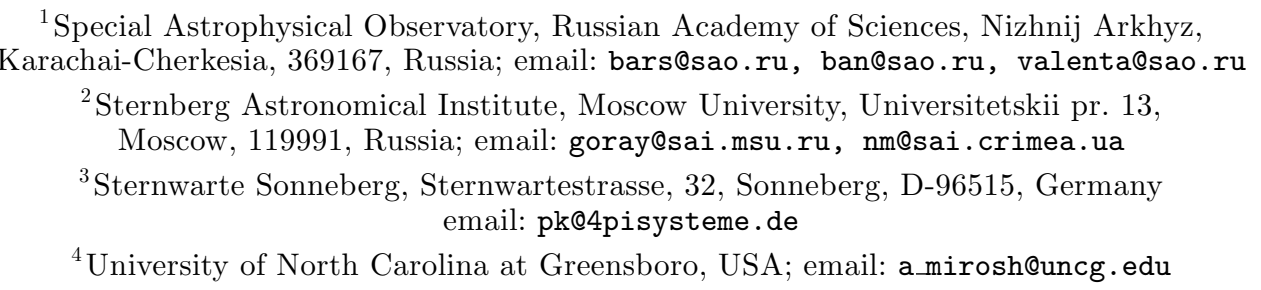

\begin{abstract}
Double mode pulsations of the B4 component in the system of CI Cam were detected. The photometric 19.4 day orbital period of CI Cam was confirmed with the plates of the Sonneberg collection for a long period of time before the unique 1998 outburst. The amplitude of the periodic component of 0.08 mag before the outburst was larger than that of 0.03 mag after the outburst.
\end{abstract}

Keywords. binaries: general, novae, cataclysmic variables, stars: oscillations (including pulsations), circumstellar matter

We present results of joint optical photometric and spectroscopic investigations of CI Cam (XTE J0421+560) for 11 years. CI Cam was an unclassified star with the B[e] phenomenon. The star was chosen due to its strong outburst in all ranges of electromagnetic spectrum in 1998. The star brightness at the peak of the outburst in optical bands exceeded its brightness in quiescence by at least a factor of 25 . This outburst has been treated by researchers as a thermonuclear runaway of hydrogen accumulated on the surface of a white dwarf, so CI Cam may be an sample of a classical nova. Such outbursts of $\mathrm{B}[\mathrm{e}]$ stars have been observed neither in the Galaxy nor in neighboring galaxies yet.

Our investigations have revealed the following properties of CI Cam. The system consists of a B4III-V star and a white dwarf on an eccentric $(\mathrm{e}=0.62)$ orbit with the period of 19.407 day. The white dwarf appears with Doppler shifts of the HeII $4686 \AA$ emission line (Barsukova et al. 2006). CI Cam demonstrates different types of optical variability including rapid intranight variations and slow quasi-periodic changes on a timescale of several years. We carried out an extensive photometric CCD monitoring (3511 images in the $\mathrm{V}$ band) and revealed pulsations of the B-type companion of CI Cam in quiescence with the amplitude up to $0^{m} .07$ (Goranskij \& Barsukova 2009). These pulsations are similar to those of classical Be stars (De Cat 2002). Two waves with periods of $0^{d} .4152$ $\left( \pm 0^{d} .0007\right)$ and $0^{d} .2667\left( \pm 0^{d} .0003\right)$ were dominating with full amplitudes of $0^{m} .019$ and $0^{m} .017$, correspondingly. There is an evidence of a resonance between these waves.

We studied also secular and orbital light variations of CI Cam using numerous digitized plates of the Sonneberg Observatory plate collection obtained between 1935 and 1967. We have confirmed the 19.4 day orbital period of CI Cam before the outburst. The amplitude of the periodic component before the outburst (Fig. 1a) is equal to 0.08 mag. After the outburst the amplitude was smaller than before the outburst (0.03 mag) (Fig. 1b). 

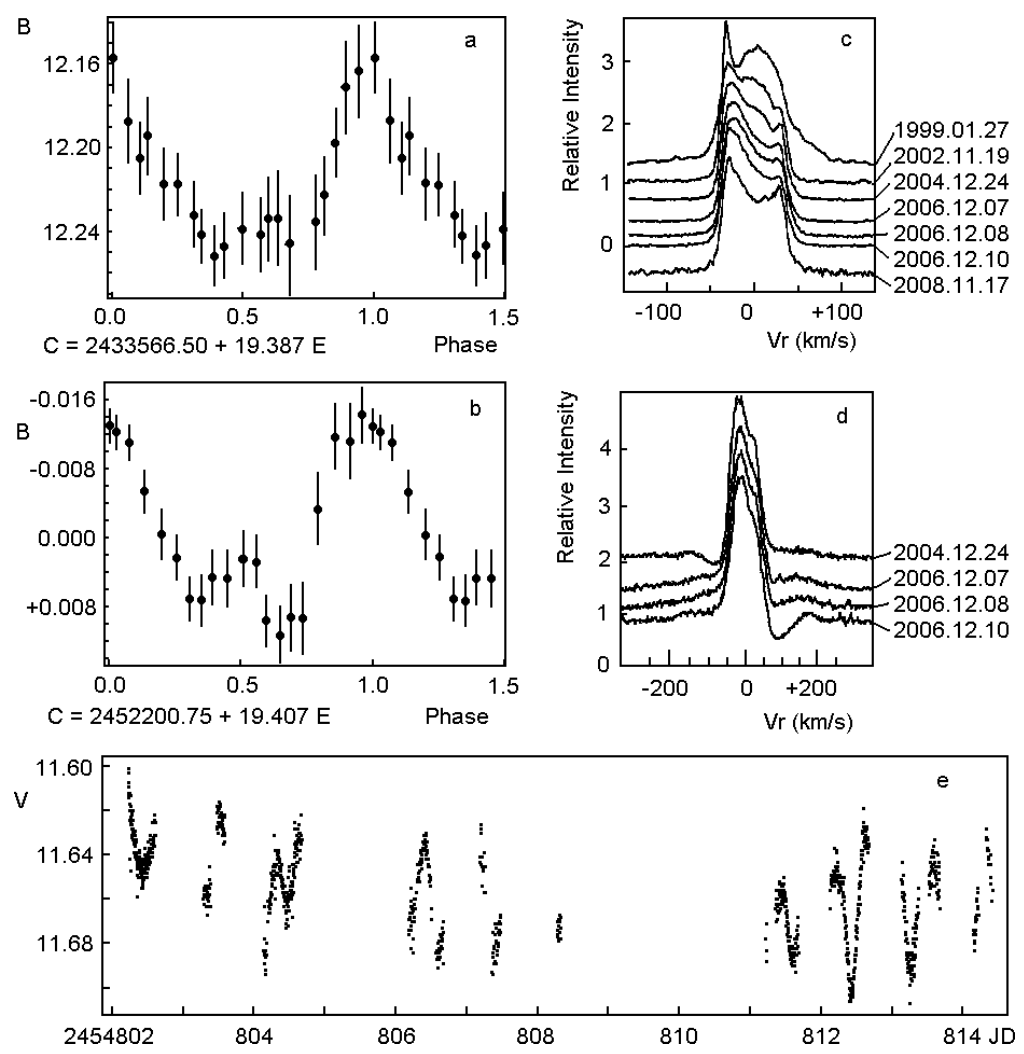

Figure 1. (a) The mean light curve of CI Cam in the B band before the outburst derived from the Sonneberg plates between 1935 and 1967. (b) The mean light curve after the outburst based on photoelectric B-band observations at the SAI Crimean Station. (c) Profile variations in the [NII] $5755 \AA$ emission line after the outburst. (d) Examples of direct and reverse P Cyg profiles of the He I $4713 \AA$ emission line in the spectrum of CI Cam at a high spectral resolution. (e) Pulsations in the $\mathrm{V}$ band.

We analyzed 19 echelle spectra (resolution $F W H M=0.08-0.21 \AA$ ) taken with the Russian 6-m telescope BTA (LYNX and NES spectrographs), and other telescopes obtained from 1999 to 2008. We found that line profiles of Fe II and [N II] emission lines in these spectra shift with time. Radial velocities of these lines do not correlate with the phase of the 19.4 day orbital period, and this shift may be due to gravitational effect from a hypothetical third massive companion (Barsukova et al. 2007). We also found temporal variations in emission line profiles. Fig. 1c shows a fading low-velocity component in the [N II] line profiles that is probably connected with dissipation of erupted matter after the outburst. Variations of He I $4713 \AA$ line profiles (Fig. 1d) may be caused by pulsations of the B-type star. A light curve showing the B star pulsations is presented in Fig. 1e.

\section{References}

De Cat, P. 2002, in: C. Aerts, T. R. Bedding, \& J. Christensen-Dalsgaard (eds.), IAU Colloq. 185: Radial and Nonradial Pulsationsn as Probes of Stellar Physics, ASP-CS 259, p. 196

Barsukova, E. A., Borisov, N. V., Burenkov, A. N., Goranskii, V. P. et al. 2006, Astron. Rep., 50, 664

Barsukova, E.A., Klochkova V.G., Panchuk V.E., Yushkin, M. V. et al. 2007, ATel, 1036

Goranskij, V. P. \& Barsukova, E. A. 2009, Astrophysical Bulletin, 64, 50 